\title{
Open-shell Donors and Closed-shell Acceptors in Organic Solar Cells
}

Zhongxin Chen, ${ }^{1}$ Yuan Li, ${ }^{* 1}$ Wenqiang Li ${ }^{1}$ Weiya Zhu, ${ }^{1}$ Miao Zeng, ${ }^{1}$ Jingwen Wang, ${ }^{2}$ Xiaojin Zhang, ${ }^{1}$ Xiaobin Peng, ${ }^{1}$ Tian Du, ${ }^{3}$ Yunfeng Deng, ${ }^{3}$ Ren Zhang, ${ }^{4}$ Gongqiang

$$
\mathrm{Li}^{4}{ }^{4} \text { Jianhui Hou }{ }^{* 2} \text { and Fei Huang }{ }^{* 1}
$$

1 State Key Laboratory of Luminescent Materials and Devices, Institute of Polymer Optoelectronic Materials and Devices, South China University of Technology, Guangzhou, 510640, P. R. China.

2 Beijing National Laboratory for Molecular Sciences, State Key Laboratory of Polymer Physics and Chemistry, CAS. Research/Education Center for Excellence in Molecular Sciences, Institute of Chemistry, Chinese Academy of Sciences, Beijing 100190, P. R. China

3 Tianjin Key Laboratory of Molecular Optoelectronic Science, Tianjin. University and Collaborative Innovation Center of Chemical Science and Engineering (Tianjin), Tianjin 300072, P. R. China.

4 Institute of Advanced Materials, Nanjing Tech University, Nanjing 211816, P. R. China.

*Correspondence author: celiy@scut.edu.cn, hjhzlz@iccas.ac.cn, msfhuang@scut.edu.cn 


\begin{abstract}
The active materials of organic solar cells are widely recognized to show closed-shell singlet ground state and their electron spin resonance signals are attributed to the defects and impurities. Herein, we disclose the inherent open-shell singlet ground state of donors and the closed-shell structure of acceptors via the combination of variable temperature NMR, electron spin resonance, superconducting quantum interference device and theoretical calculation, providing a new perspective to understand the intrinsic molecular structure in organic solar cells.
\end{abstract}

\title{
Introduction
}

Organic solar cell (OSC) is a promising photovoltaic technology due to its flexibility, low-cost, light weight, solution processability and high-throughput roll-to-roll production. ${ }^{1,2}$ During the past several decades, the advances mainly rely on the development of bulk heterojunction (BHJ) active materials consisting of the nanostructured electron donor and electron acceptor components. ${ }^{2,3}$ Nowadays, the innovations in efficient materials led by donors, ${ }^{4,5}$ non-fullerene acceptors, ${ }^{6}$ morphology optimization, ${ }^{7}$ interface engineering and tandem cell strategy ${ }^{8}$ have pushed the power conversion efficiency to over $18 \% .^{5}$ In addition to the pursuit of highefficiency materials, the improvement of material stability is equally important for the practical application for OSCs. ${ }^{9}$ Given this importance, the intrinsic degradation pathways of the active materials under realistic operation conditions should be well understood. It was reported that the light-induced dimerization of the fullerene acceptors such as $\mathrm{PC}_{61} \mathrm{BM}$ resulted in the rapid degradation of the OSCs. ${ }^{10}$ Recent work demonstrated that the $\pi$-conjugated polymer donors and non-fullerene acceptors also show the similar light-induced crosslinking reaction with that of fullerenes, which results in the decay of the photovoltaic efficiency. ${ }^{11}$ This so-call burn-in effect of OSCs is correlated with the generation of active radical centers in the molecular backbones of donors and acceptors. However, the origin of the radicals in active materials has not been well understood. The previous reports have attributed the radical species and the associated ESR response detected in donors and acceptors to the structural defects, impurities, ${ }^{12,} 13$ traps, ${ }^{14}$ or radical cation/anions. ${ }^{15}$ In our previous work, we demonstrated that the narrow bandgap donor-acceptor type organic semiconductors commonly showed electron spin resonance (ESR) signals and proposed the mechanism of quinoid-diradical resonance structure. ${ }^{16}$ 
To better reveal the origin of radicals of donors and acceptors in OSCs, in the current work, we correlated the relationship between the electronic ground state and the materials. We found that the donors commonly possessed distinct open-shell radical character while the acceptors showed none of radical character in their ground states. The significantly different radical character between donors and acceptors is closely corelated with their molecular conformations and electronic structures. This work provided a novel understanding of the intrinsic electronic structure of active materials in OSCs.
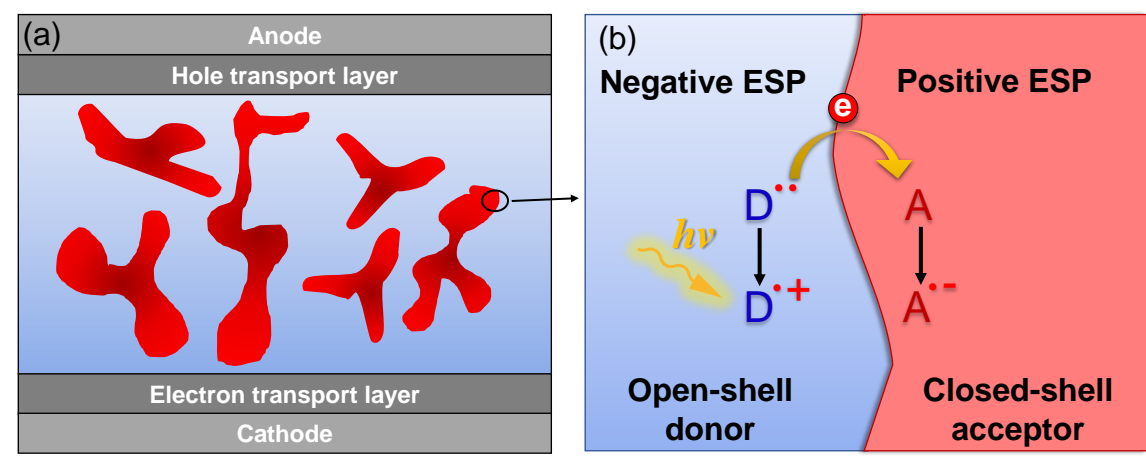

Figure 1. (a, b) Diagram of the formation and dissociation of excitons occur in the donor/acceptor interface. The diradical character of open-shell donors show negative average ESP values, while the acceptors show positive average values. The formation of intermolecular electric field facilitates the electron transfer from donor to acceptor. The donor and acceptor domains are drawn as blue and red colors, respectively.

\section{Results and discussion}

To establish the correlations between donors/acceptors and radical character, we focus on the prototypical materials commonly adopted in active layers of OSCs and investigate their electronic ground-state structures. Electronic spin resonance (ESR) spectroscopy ${ }^{17}$ was conducted to the powder samples which were carefully sealed in ESR tubes. For donors, we investigated the acceptor-donor-acceptor (A-D-A) type narrow bandgap small molecules including BTR, ${ }^{18}$ DI3T, ${ }^{19}$ DI3T-2F, ${ }^{19}$ DPPEZnPTEH, ${ }^{20}$ (Fig. 2a, Fig. S1) and the polymers including PTB7-Th, ${ }^{21} \mathrm{PM} 6,{ }^{22}$ and D1 $8^{5}$ (Fig. S1). All the donors including small molecules and polymers show pronounced ESR signal with similar $g$ values (2.0035-2.0040) (Fig. 2b), which is closed to the value of carbon-based free electron, demonstrating the presence of paramagnetic radicals in donors. ${ }^{23}$ For acceptors, the NFAs including ITIC, ${ }^{24}$ IT-4F, ${ }^{25}$ IDIC, ${ }^{26}$ IEICO-4F, ${ }^{27}$ IDTBR, ${ }^{28}$ and $\mathrm{Y}^{22}$ were adopted as model compounds (Fig. S1) to illustrate their ground-state electronic structures. (Fig. 2c) Totally different from the donors, all the 
measured acceptors exhibited none of ESR response (Fig. 2d). The calculated diradical index $y_{0}$ and tetraradical character index $y_{1}$ of the donors and acceptors are also corroborated with the ESR measurements. (Table 1) The $y_{0}$ of donors are higher than that of acceptors, indicating the contribution of Q-D structure and the significant diradical character of efficient donors. The different quinoid-diradical character between donors and acceptors can be reflected by the molecular electrostatic potential (ESP) distribution, which denotes the feature of electron density distribution. ${ }^{29}$ The donors with quinoidal character and relatively rich electron density show the negative ESP on the most part of their molecular surfaces, while the acceptors with relatively even electron density distribution show positive average ESP (Fig. S4). ${ }^{29,30}$
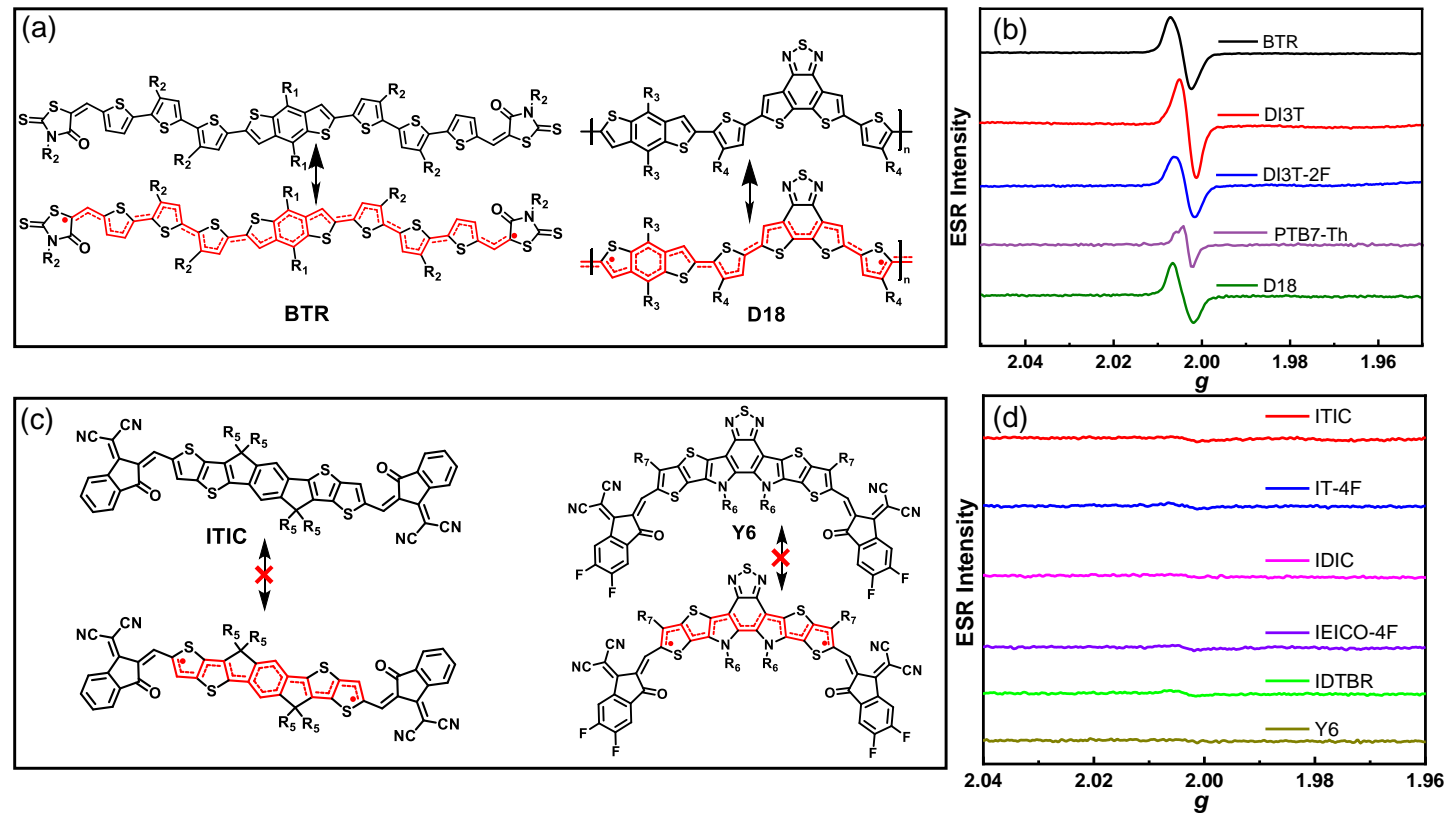

Figure 2. (a) Molecular structures of BTR and D18 and the formation of quinoid-diradical structure. Their open-shell diradical resonance structures are presented. $\mathrm{R}_{1}=2$-(2-ethylhexyl)-3-hexyl-5thienyl, $\mathrm{R}_{2}=n$-hexyl, $\mathrm{R}_{3}=2$-(2-ethylhexyl)-3-fluoro-5- thienyl, $\mathrm{R}_{4}=$ 2-butyloctyl. (b) ESR spectra of the donors. (c) Molecular structures of the non-fullerene acceptors. $R_{5}=4$-hexylphenyl, $R_{6}=2$ ethylhexyl, $\mathrm{R}_{7}=n$-undecyl. (d) ESR spectra of the non-fullerene acceptors.

Table 1. Calculated diradical character index $\left(y_{0}\right)$ and tetraradical character index $\left(y_{I}\right)$ of the donors and acceptors calculated with PUHF/6-31G $(d, p)$.

\begin{tabular}{cccccccccc}
\hline & BTR & DI3T & DI3T-2F & $\begin{array}{c}\text { PTB7-Th } \\
(\mathrm{n}=4)\end{array}$ & ITIC & IT-4F & Y6 & IDIC & IDI \\
\hline$y_{0}$ & 0.320 & 0.301 & 0.300 & 0.324 & 0.294 & 0.295 & 0.257 & 0.279 & 0.502 \\
$y_{1}$ & 0.226 & 0.202 & 0.201 & 0.232 & 0.163 & 0.163 & 0.132 & 0.144 & 0.323 \\
\hline
\end{tabular}


Radicals have a shielding effect on the hydrogen NMR spectrum, and it is usually supplemented with ESR to verify the presence of paramagnetic radicals. ${ }^{31,32}$ For the high-performance donor BTR in $\mathrm{C}_{2} \mathrm{D}_{2} \mathrm{Cl}_{4}$, the peaks in aromatic region become broadened upon heating from 293 to 393 K (Fig. 3a and Fig. 3b), while the protons on the alkyl chains do not show broadened NMR peaks (Fig. S2). The broadened and blurred peaks in NMR is the typical characteristic of triplet radicals (Fig. 3c). ${ }^{33}$ The tendence of the NMR peaks of the protons on BDT core and the adjacent thiophenes (b, $\mathrm{f}, \mathrm{g}$ protons,) to broadened is more obvious compared with the other protons, indicating the large spin density on the BDT core. Variable-temperature ESR spectroscopy and superconducting quantum interference device (SQUID) measurements were also conducted on BTR to provide a convincing evidence. The ESR signal increased with the increase of temperature (Fig. 3d) due to the indicated the population of triplet radicals. ${ }^{31}$ From the SQUID measurement, the $\chi_{\mathrm{M}} \cdot T$ exhibited a positive corelation with the $T$ (Fig. 3e). Fitting the data with Bleaney-Bowers equation gave the $\Delta E_{\mathrm{ST}}$ of -312.9 $\mathrm{K}$, indicating the narrow singlet-triplet energy gap. ${ }^{34}$ The SQUID measurement on polymer donor PTB7-Th was also conducted to provide a convincing evidence. The $\Delta E_{\mathrm{ST}}$ obtained by fitting the data with Bleaney-Bowers equation is $-1920.51 \mathrm{~K}$. (Fig. S3) The significant ESR response, enhanced ESR signal and broadened NMR spectra upon heating, as well as the small singlet-triplet splitting demonstrated the open-shell diradical character with singlet ground state $\left(\mathrm{S}_{0}\right)$ and thermally accessible triplet state $\left(\mathrm{T}_{\mathrm{t}}\right)$ of the donors (Fig. 3f). ${ }^{34,35}$

This strikingly different result between donors and acceptors has a close relationship to their molecular structure and ground-state geometric conformation. For donors, the embedding of quinoid-like backbones, and the planar geometry are essential to lower their bandgaps and improve charge mobility as well as the crystallization capability. ${ }^{36}$, ${ }^{37}$ On the other hand, these factors also contribute to the conversion from close-shell aromatic form to quinoid-diradical form as depicted in Fig. 2a. Taking BTR as an example, the insertion of benzodithiophene (BDT) core with proquinoidal character promoted the resonant conversion to diradical forms, as demonstrated by the increase of bond length alternation (BLA) along the conjugated backbone according to the calculated geometric parameters (Fig. S5). The planar geometric structure of BTR is also advantageous of the delocalization and stabilization of radicals. In contrast, the acceptors such as ITIC and Y6 have highly fused conjugated cores, which indicated that the quinoid-diradical form are less thermodynamically favourable due to the 
preferred aromaticity of the fused cores, inhibiting the transition of aromatic form into the quinoid-diradical structure. The weaker quinoidal character of ITIC is evident from a reduced BLA in the fused core, where each of the thienyl rings have almost equal bonds and a very small BLA (Fig. S5) according to the DFT calculations of ITIC. In addition, the calculated NICS $_{\text {iso }}(1)$ of ITIC core were relatively higher than that of the BTR core, indicating its higher aromatic character of ITIC (Fig. S5).
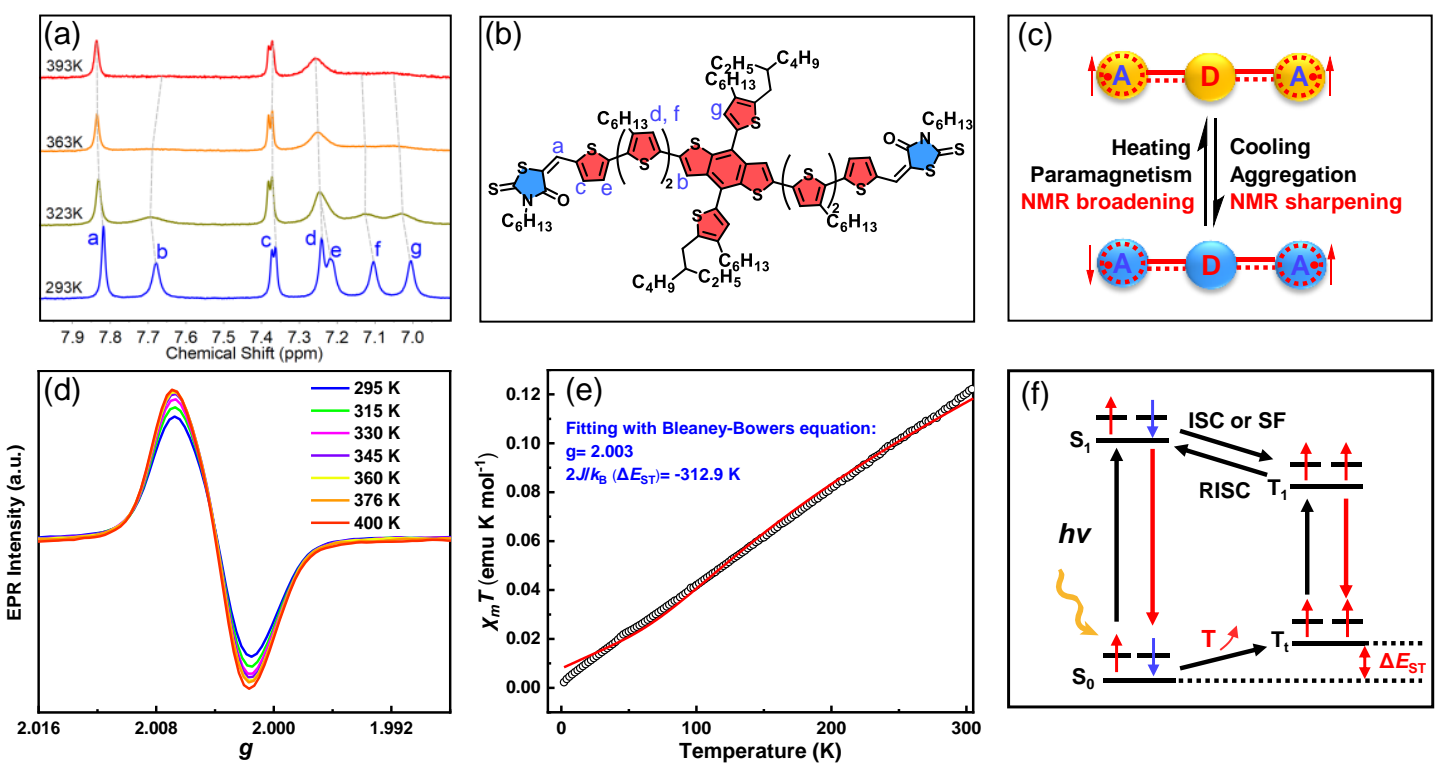

Figure 3. (a) Variable-temperature ${ }^{1} \mathrm{H}$ NMR spectra of BTR. (b) Molecular structure of BTR. The donor and acceptor units are filled with red and blue, respectively. (c) Thermally accessible triplet state of acceptor-donor-acceptor (A-D-A) molecules. The elevate of temperature induces the generation of paramagnetic radicals, which results in the broadening NMR spectra, while the decrease of temperature results in the NMR sharpening because of strong aggregation. (d) Variabletemperature ESR spectra of BTR. (e) The product of magnetic susceptibility and temperature versus temperature $\left(\chi_{\mathrm{m}} T-T\right)$ of BTR obtained via SQUID. (f) Diagram of the singlet ground state $\left(\mathrm{S}_{0}\right)$ and thermally accessible triplet state $\left(\mathrm{T}_{\mathrm{t}}\right)$, as well as the first singlet $\left(\mathrm{S}_{1}\right)$ and triplet excited state $\left(\mathrm{T}_{1}\right)$ of open-shell donor molecules. The parameters of ISC, RISC and SF imply for the intersystem crossing, reverse intersystem crossing, and singlet fission. The parameter of $\Delta E_{\mathrm{ST}}$ represents the energy gap between $\mathrm{S}_{0}$ and $\mathrm{T}_{\mathrm{t}}$.

In order to understand the structure-property relationship of the ESR-silent NFAs, we studied the quinoid-diradical characteristic of the acceptor IDIC and its indandioneterminated quinoidal analogue ${ }^{38}$, IDI. Acceptor IDIC (Fig. 4a) has a nearly silent ESR response, however, the quinoidal IDI (Fig. 4b) shows a significant ESR intensity (Fig. 4c). This is also corroborated by the calculated higher diradical character of IDI than IDIC. The photovoltaic performance of the devices based on these two acceptor-like 
molecules differ significantly (Fig. 4d, Fig. S6 and Table S1) with the same donor PTB7-Th. The active layer of PBT7-Th/IDIC devices show a power conversion efficiency (PCE) of $5.09 \%$ with a $J_{\mathrm{sc}}$ of $12.60 \mathrm{~mA} / \mathrm{cm}^{2}$, however, the devices based on PTB7-Th/IDI as active layer show an extremely low PCE of $0.11 \%$, implying that IDI is a poor acceptor.
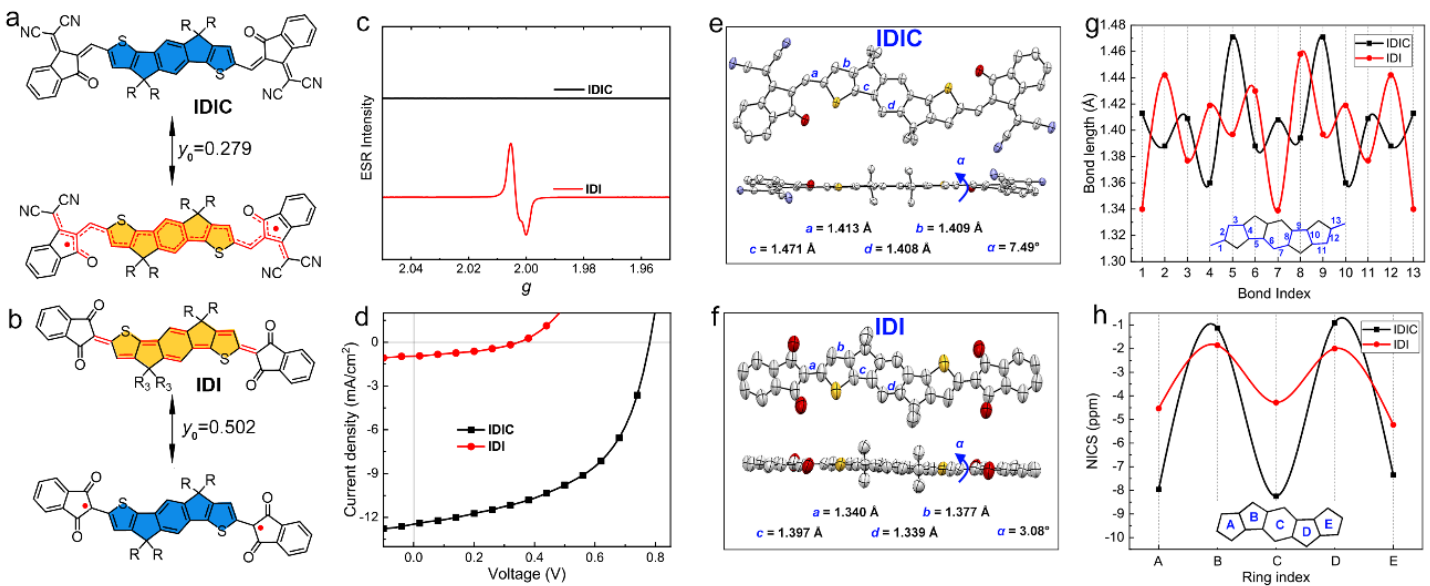

Figure 4. (a, b) The resonance structures of A-D-A OSCs (R group: 4-hexylphenyl). (c, d) The ESR spectra and $J-V$ curves of the OPV devices with PTB7-Th as donor, and IDI and IDIC as acceptors, respectively. (e, f) The ORTEP images according to X-ray crystallography. Hydrogen atoms are omitted and the long alkyl chain are replaced with methyl group for clarity. $(\mathrm{g})$ The BLA curves of the IDT core. (h) The NICS iso $_{\text {(1) }}$ curves of the IDT core

To further disclose the underlying mechanism for the significant difference of ESR response of IDIC and IDI, the chemical entities, and electronic structures of IDIC and IDI are carefully compared and investigated according to single-crystal X-ray diffraction studies and DFT calculations. The crystals of IDIC and IDI are reported in the previous works ${ }^{39,40}$ (Fig. 4e, 4f). The lengths of bonds $a, b, c, d$ of IDI are observed to be shorter than those of the moieties are covalently linked by double bonds. The quinoidal molecule has a high degree of electronic coherence along the $\pi$-conjugated backbone, which can be attributed to the direct coupling of the strong electronwithdrawing indandione unit and recovery of the aromaticity in the IDT core. This is also supported by NICS $_{i s o}(1)$ values (Fig. $\mathbf{4 g}$ ) of the cores of IDI and IDIC, where a reduced NICS $_{i s o}(1)$ is observed in the IDI core than IDIC (Fig. 4h). Moreover, the aromaticity of IDI core increases from singlet to triplet, while that of IDIC decreases, which suggests that the triplet state in IDI is thermodynamically stabilized (Fig. S7). The AICD plots (Fig. S8) indicate a weak counter clockwise paratropic ring current in the IDI core, with strong clockwise diatropic ring current at the external benzenoid 
rings, while strong diatropic ring current is visible in the IDIC core. These results indicate the apparent quinoid-diradical electronic structure of IDI, while the aromatic form is more thermodynamically stable for the IDIC acceptor.

To further investigate the quinoid-diradical property of NFAs and the influence of radicals on the performance of organic solar cell, we synthesized a diketopyrrolopyrrole (DPP) -based small molecule DPPC-IC4F and studied the structure-property relationship (Fig. 5a). In previous work, we reported the distinct diradical character of DPP-based small molecules because of the quinoidal property of the DPP core, and the diradical character can be further enhanced as the D-A characteristic enhanced. ${ }^{16}$ For DPPC-IC4F, the insertion of strong D and A units contributed to the extended absorption tail to over $1100 \mathrm{~nm}$, with a narrow bandgap of $1.10 \mathrm{eV}$ (Fig. 5b). The highest occupied molecular orbital (HOMO) and lowest unoccupied molecular orbital (LUMO) of DPPC-IC4F were calculated to be -4.92 and $-3.86 \mathrm{eV}$, respectively, according to the cyclic voltammetry measurement (Fig. 5c). DPPC-IC4F showed a broadened ${ }^{1} \mathrm{H}$ NMR peaks (from 6.5-9.0 ppm) at room temperature, and the proton peaks become even broadened combining with the increment of temperature, indicating the population of triplet species at relatively high temperature (Fig. 5a). DPPC-IC4F exhibited a strong ESR response at room temperature with a $g$-factor of 2.0037, indicating the formation of open-shell radical species. The ESR signal of DPPC-IC4F enhanced as the temperature is increased, which denoted the singlet ground state as well as the thermally accessible triplet state (Fig. 5d). SQUID measurements were conducted to further demonstrate the ground-state electronic structure of DPPC-IC4F. The $\chi_{\mathrm{M}} \cdot T-T$ curve exhibited a typical characteristic of singlet-to-triplet transition (Fig. 5e) ${ }^{33,41}$ Fitting the data with Bleaney-Bowers equation gave the $\Delta E_{\mathrm{ST}}$ of $-894.6 \mathrm{~K}$. To investigated the photovoltaic performance, PTB7-Th/DPPC-IC4F blend was prepared as the active layer in OSC device. The OSCs exhibited a poor efficiency of $0.57 \%$ (Fig. S9). We proposed that in the photophysical process of OSCs, the radical acceptor may act as the trap to hinder the charge transfer, which results in the poor short-circuit current and photovoltaic performance. 

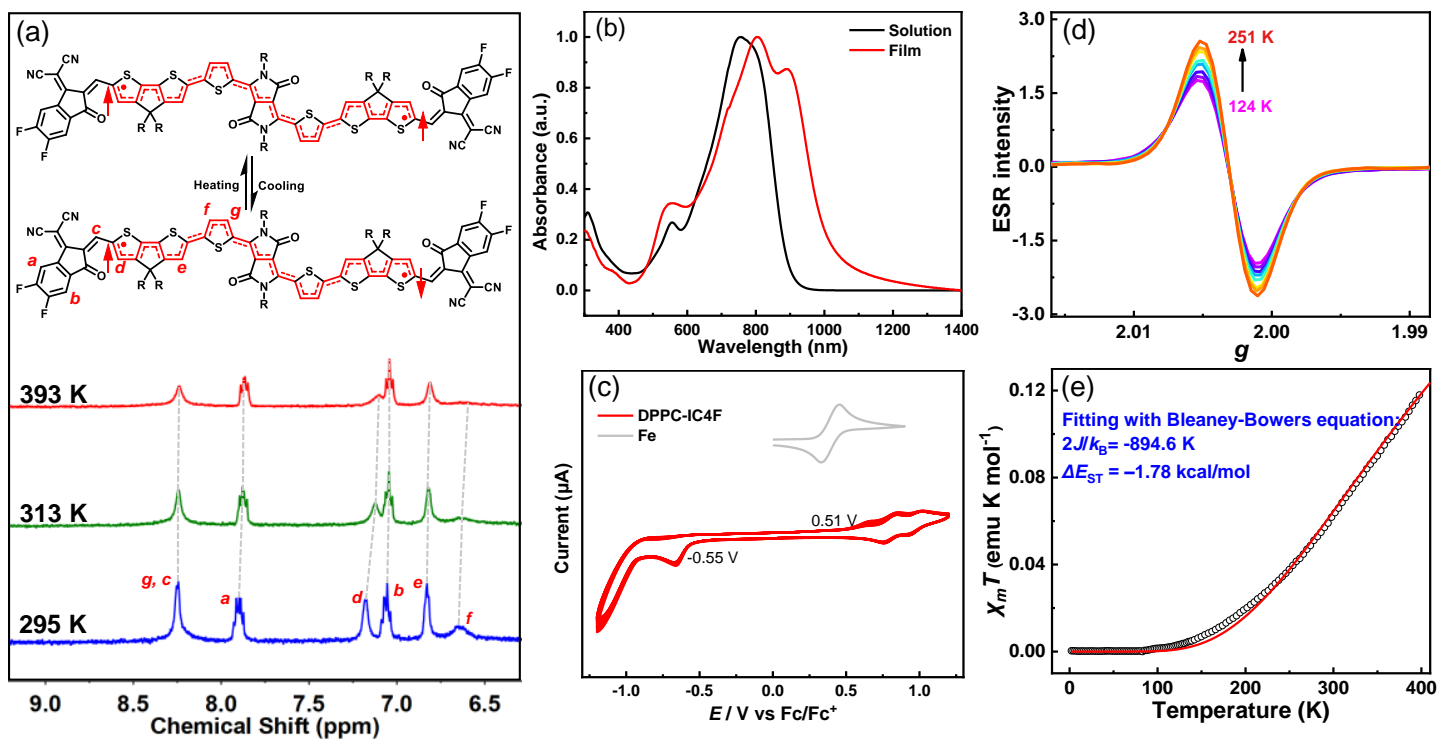

Figure 5. (a) Molecular structure and variable temperature NMR spectra from 295 to $393 \mathrm{~K}$ of DPPC-IC4F, (b) UV-vis-NIR spectra of DPPC-IC4F in chloroform and in thin film, respectively. (c) Cyclic voltammetry curve of DPPC-IC4F in dichloromethane (30 cycles), with ferrocene as the internal standard. (d) Variable temperature ESR spectra of DPPC-IC4F from 124 to $251 \mathrm{~K}$. (e) $\chi_{M} T$ $T$ plot and Bleaney-Bowers fitting curves of DPPC-IC4F.

Based on the above discussions, we proposed that the radical character may play an important role for the design of high-performing donors and acceptors. (Fig. 1a, 1b). For donors, the quinoid-diradical structure is ubiquitous. However, efficient donors should be constructed to be ESR-silent or nearly ESR-silent. The donor materials without radical character can enhance the fluorescence quantum yields, exciton dissociation and device photostability. ${ }^{16}$ Geng et al. designed efficient donors based on polythiophenes exhibiting PCEs of 10-12\%. ${ }^{42}$ The introduction of ester group into the side chain contributed to lowering HOMOs, and thus reducing the formation of quinoid-diradical structure. ${ }^{16,34}$ The efficient donor polymers based on B-N covalent bond yielded high PCEs of over $16 \%$ when cooperating with Y $6 .{ }^{43}$ The insertion of B$\mathrm{N}$ unit also reduced the diradical character of the polymers and contributed to the high solar cell performance. For acceptors, the incorporation of fused aromatic rings contributes to the robust ICT effect and narrow bandgap, and also improves the stabilization of closed-shell structure. The quinoidal character may act as traps in the exciton dissociation process and should be avoided. We proposed that the cooperation 
of donors and acceptors with silent ESR response is the design strategy for the efficient OSCs.

\section{Conclusions}

In summary, we report that the donors of active material in OSCs widely exhibit intrinsic open-shell character while the high-performance NFAs show closed-shell with none of ESR signal. The radical characters of donors and acceptors are closely related to their molecular structures and configurations. Moreover, fine tailoring of the molecular structure will effectively modulate the radical characters of donors/acceptors, which can be demonstrated by the obvious changes of chemical/physical properties in OSCs. Our studies provided a novel and important guideline for the design of donors and acceptors in OSCs.

\section{Materials and methods}

Continuous wave X-band EPR spectra of powder samples were recorded on Bruker ELEXSYS-II E500 CW-EPR spectrometer. The molar quantity of the material was 0.02 mmol. The spectra were recorded at room temperature at a signal attenuation of $20 \mathrm{~dB}$ in quartz ESR standard quality tubes with an outer diameter of $5 \mathrm{~mm}$ from Beijing Magnetic measurements were carried out on a Quantum Design MPMS XL SQUID Magnetometer. The samples were prepared by placing $15 \mathrm{mg}$ of PTB7-Th and $20 \mathrm{mg}$ of BTR in a polycarbonate capsule. Prior to measurement, the background of the empty capsule was recorded. Temperature-dependent data was collected from $T=2.5-300 \mathrm{~K}$ at an applied field of 5000 Oe. The susceptibility data were corrected for the diamagnetism of the sample holder and intrinsic diamagnetism of the sample. The magnetic susceptibility of BTR and PTB7-Th was fit using the equation:

$$
\chi=\frac{2 N g^{2} \mu_{\beta}^{2}}{k T}\left[\frac{1}{3+e^{-2 J / k T}}\right](1-\rho)+\frac{N g^{2} \mu_{\beta}^{2}}{2 k T} \rho+T I P(1-\rho)
$$

where $\rho$ is the fraction of $S=1 / 2$ impurity and TIP is the temperature independent paramagnetism due to the small energy gap between singlet state and triplet state.

The Gaussian 16 program package ${ }^{44}$ was used for the geometry optimizations of the low bandgap OSCs using hybrid density functional, B3LYP ${ }^{45,46}$. LANL2TZ basis set was used for $\mathrm{Zn}$ with associated effective core potentials ${ }^{47}$ and 6-31G $(d, p)$ basis set $^{48}$ 
used for other atoms. The local minima of the optimized structures were verified with frequency calculations. All conditions of the geometry optimizations were used as defaults. For all the low bandgap OSCs, the side chains were truncated to $-\mathrm{CH}_{3}$. The diradical $\left(y_{0}\right)$ and tetraradical $\left(y_{1}\right)$ character were calculated with the spin-projected unrestricted Hartree-Fock method using Yamaguchi's formula ${ }^{49}$ at $\mathrm{UHF} / 6-31 \mathrm{G}(d, p)$ level of theory and basis set:

$$
y_{i}=1-\frac{2 T_{i}}{1+T_{i}^{2}} ; \quad T_{i}=\frac{n_{H O N O-i}-n_{L U N O+i}}{2}
$$

where, $i=0,1 ; T_{i}$ is the overlap between the two corresponding orbitals; $n_{\text {HONO }}$ and $n_{\text {LUNO }}$ are the occupation numbers of the highest occupied natural orbital and the lowest unoccupied natural orbital, respectively. Nucleus independent chemical shift (NICS $\left._{i s o}(1)\right)$ was computed at $1 \AA$ above the rings plane with gauge-independent atomic orbital (GIAO) method. ${ }^{50}$ The optical properties were modeled with time-dependent density functional theory (TDDFT) at PCM/B3LYP/6-31G $(d, p)$ level of theory and basis set and chloroform was used as an implicit solvent. ${ }^{51}$ AICD (anisotropy of the induced current density) plots were generated by the method developed by Herges et $a .^{52}$

The ESP and intermolecular interaction were performed by Gaussian 09 at B3LYP/6$31 \mathrm{G}(\mathrm{d}, \mathrm{p})$ level. The binding energies were corrected by DFT-D3. The excited-state calculations were obtained by time-dependent DFT at cam-B3LYP/6-31G $(d, p)$ S5 level. The analysis of ESP on each atom, area distributions, electron-hole distance, and attractive energy was conducted by a wavefunction software Multiwfn.

\section{Conflict of interest}

There are no conflicts to declare.

\section{Acknowledgements}

The authors acknowledge the financial support of the Natural Science Foundation of China (No. 51973063), Tip-top Scientific and Technical Innovative Youth Talents of Guangdong Special Support Program (2019TQ05C890), the Pearl River S\&T Nova Program of Guangzhou (201710010194), the Basic and Applied Basic Research Major 
Program of Guangdong Province (No. 2019B030302007), National Key Research and

Development Program of China (No. 2019YFA0705900) funded by MOST.

\section{References}

1. Brabec, C. J.; Gowrisanker, S.; Halls, J. J.; Laird, D.; Jia, S.; Williams, S. P., Polymer-fullerene bulk-heterojunction solar cells. Adv. Mater. 2010, 22 (34), 38393856.

2. Heeger, A. J., 25th anniversary article: Bulk heterojunction solar cells: understanding the mechanism of operation. Adv Mater 2014, 26 (1), 10-27.

3. Yu, G.; Gao, J.; Hummelen, J. C.; Wudl, F.; Heeger, A. J., Polymer Photovoltaic Cells: Enhanced Efficiencies via a Network of Internal Donor-Acceptor Heterojunctions. Science 1995, 270 (5243), 1789-1791.

4. Chen, H.; Hu, D.; Yang, Q.; Gao, J.; Fu, J.; Yang, K.; He, H.; Chen, S.; Kan, Z.; Duan, T.; Yang, C.; Ouyang, J.; Xiao, Z.; Sun, K.; Lu, S., All-SmallMolecule Organic Solar Cells with an Ordered Liquid Crystalline Donor. Joule 2019, 3 (12), 3034-3047.

5. Liu, Q.; Jiang, Y.; Jin, K.; Qin, J.; Xu, J.; Li, W.; Xiong, J.; Liu, J.; Xiao, Z.; Sun, K.; Yang, S.; Zhang, X.; Ding, L., 18\% Efficiency organic solar cells. Sci. Bull. 2020, 65 (4), 272-275.

6. Cheng, P.; Li, G.; Zhan, X.; Yang, Y., Next-generation organic photovoltaics based on non-fullerene acceptors. Nat. Photonics 2018, 12 (3), 131-142.

7. Campoy-Quiles, M.; Ferenczi, T.; Agostinelli, T.; Etchegoin, P. G.; Kim, Y.; Anthopoulos, T. D.; Stavrinou, P. N.; Bradley, D. D.; Nelson, J., Morphology evolution via self-organization and lateral and vertical diffusion in polymer:fullerene solar cell blends. Nat. Mater. 2008, 7 (2), 158-164.

8. Meng, L.; Zhang, Y.; Wan, X.; Li, C.; Zhang, X.; Wang, Y.; Ke, X.; Xiao, Z.; Ding, L.; Xia, R.; Yip, H.-L.; Cao, Y.; Chen, Y., Organic and solution-processed tandem solar cells with 17.3\% efficiency. Science 2018, 361 (6407), 1094.

9. Cheng, P.; Zhan, X., Stability of organic solar cells: challenges and strategies. Chem. Soc. Rev. 2016, 45 (9), 2544-2582.

10. Distler, A.; Sauermann, T.; Egelhaaf, H.-J.; Rodman, S.; Waller, D.; Cheon, K.-S.; Lee, M.; Guldi, D. M., The Effect of PCBM Dimerization on the Performance of Bulk Heterojunction Solar Cells. Adv. Energy Mater. 2014, 4 (1), 1300693.

11. Yamilova, O. R.; Martynov, I. V.; Brandvold, A. S.; Klimovich, I. V.; Balzer, A. H.; Akkuratov, A. V.; Kusnetsov, I. E.; Stingelin, N.; Troshin, P. A., What is Killing Organic Photovoltaics: Light-Induced Crosslinking as a General Degradation Pathway of Organic Conjugated Molecules. Adv. Energy Mater. 2020, 10 (7), 1903163. 12. Susarova, D. K.; Piven, N. P.; Akkuratov, A. V.; Frolova, L. A.; Polinskaya, M. S.; Ponomarenko, S. A.; Babenko, S. D.; Troshin, P. A., ESR spectroscopy as a powerful tool for probing the quality of conjugated polymers designed for photovoltaic applications. Chem. Commun. 2015, 51 (12), 2239-2241.

13. Frolova, L. A.; Piven, N. P.; Susarova, D. K.; Akkuratov, A. V.; Babenko, S. D.; Troshin, P. A., ESR spectroscopy for monitoring the photochemical and thermal degradation of conjugated polymers used as electron donor materials in organic bulk heterojunction solar cells. Chem. Commun. 2015, 51 (12), 2242-2244. 
14. Schafferhans, J.; Baumann, A.; Deibel, C.; Dyakonov, V., Trap distribution and the impact of oxygen-induced traps on the charge transport in poly(3hexylthiophene). Appl. Phys. Lett. 2008, 93 (9), 093303.

15. Li, S.; Yuan, N.; Fang, Y.; Chen, C.; Wang, L.; Feng, R.; Zhao, Y.; Cui, H.; Wang, X., Studies on the Bridge Dependence of Bis(triarylamine) Diradical Dications: Long-Range $\pi$-Conjugation and $\pi$ - $\pi$ Coupling Systems. J. Org. Chem. 2018, 83 (7), 3651-3656.

16. Li, Y.; Li, L.; Wu, Y.; Li, Y., A Review on the Origin of Synthetic Metal Radical: Singlet Open-Shell Radical Ground State? J. Phys. Chem. C 2017, 121 (15), 8579-8588.

17. Roessler, M. M.; Salvadori, E., Principles and applications of EPR spectroscopy in the chemical sciences. Chem. Soc. Rev. 2018, 47 (8), 2534-2553.

18. Sun, K.; Xiao, Z.; Lu, S.; Zajaczkowski, W.; Pisula, W.; Hanssen, E.; White, J. M.; Williamson, R. M.; Subbiah, J.; Ouyang, J.; Holmes, A. B.; Wong, W. W.; Jones, D. J., A molecular nematic liquid crystalline material for high-performance organic photovoltaics. Nat. Commun. 2015, 6, 6013.

19. Yang, Y.; Wang, K.; Li, G.; Ran, X.; Song, X.; Gasparini, N.; Zhang, Q. Q.; Lai, X.; Guo, X.; Meng, F.; Du, M.; Huang, W.; Baran, D., Fluorination Triggered New Small Molecule Donor Materials for Efficient As-Cast Organic Solar Cells. Small 2018, 14 (34), e1801542.

20. Gao, K.; Li, L.; Lai, T.; Xiao, L.; Huang, Y.; Huang, F.; Peng, J.; Cao, Y.; Liu, F.; Russell, T. P.; Janssen, R. A.; Peng, X., Deep absorbing porphyrin small molecule for high-performance organic solar cells with very low energy losses. J. Am. Chem. Soc. 2015, 137 (23), 7282-5.

21. Liao, S. H.; Jhuo, H. J.; Cheng, Y. S.; Chen, S. A., Fullerene derivative-doped zinc oxide nanofilm as the cathode of inverted polymer solar cells with low-bandgap polymer (PTB7-Th) for high performance. Adv. Mater. 2013, 25 (34), 4766-4771.

22. Yuan, J.; Zhang, Y.; Zhou, L.; Zhang, G.; Yip, H.-L.; Lau, T.-K.; Lu, X.; Zhu, C.; Peng, H.; Johnson, P. A.; Leclerc, M.; Cao, Y.; Ulanski, J.; Li, Y.; Zou, Y., Single-Junction Organic Solar Cell with over 15\% Efficiency Using Fused-Ring Acceptor with Electron-Deficient Core. Joule 2019, 3 (4), 1140-1151.

23. Abe, M., Diradicals. Chem. Rev. 2013, 113 (9), 7011-7088.

24. Lin, Y.; Wang, J.; Zhang, Z. G.; Bai, H.; Li, Y.; Zhu, D.; Zhan, X., An electron acceptor challenging fullerenes for efficient polymer solar cells. Adv. Mater. 2015, 27 (7), 1170-1174.

25. Zhao, W.; Li, S.; Yao, H.; Zhang, S.; Zhang, Y.; Yang, B.; Hou, J., Molecular Optimization Enables over 13\% Efficiency in Organic Solar Cells. J. Am. Chem. Soc. 2017, 139 (21), 7148-7151.

26. Lin, Y.; He, Q.; Zhao, F.; Huo, L.; Mai, J.; Lu, X.; Su, C. J.; Li, T.; Wang, J.; Zhu, J.; Sun, Y.; Wang, C.; Zhan, X., A Facile Planar Fused-Ring Electron Acceptor for As-Cast Polymer Solar Cells with $8.71 \%$ Efficiency. J. Am. Chem. Soc. 2016, 138 (9), 2973-2976.

27. Yao, H.; Cui, Y.; Yu, R.; Gao, B.; Zhang, H.; Hou, J., Design, Synthesis, and Photovoltaic Characterization of a Small Molecular Acceptor with an Ultra-Narrow Band Gap. Angew. Chem. Int. Ed. 2017, 56 (11), 3045-3049.

28. Holliday, S.; Ashraf, R. S.; Wadsworth, A.; Baran, D.; Yousaf, S. A.; Nielsen, C. B.; Tan, C. H.; Dimitrov, S. D.; Shang, Z.; Gasparini, N.; Alamoudi, M.; Laquai, F.; Brabec, C. J.; Salleo, A.; Durrant, J. R.; McCulloch, I., High-efficiency and airstable P3HT-based polymer solar cells with a new non-fullerene acceptor. Nat. Commun. 2016, 7, 11585. 
29. Yao, H.; Qian, D.; Zhang, H.; Qin, Y.; Xu, B.; Cui, Y.; Yu, R.; Gao, F.; Hou, J., Critical Role of Molecular Electrostatic Potential on Charge Generation in Organic Solar Cells. Chin. J. Chem. 2018, 36 (6), 491-494.

30. Yao, H.; Cui, Y.; Qian, D.; Ponseca, C. S., Jr.; Honarfar, A.; Xu, Y.; Xin, J.; Chen, Z.; Hong, L.; Gao, B.; Yu, R.; Zu, Y.; Ma, W.; Chabera, P.; Pullerits, T.; Yartsev, A.; Gao, F.; Hou, J., 14.7\% Efficiency Organic Photovoltaic Cells Enabled by Active Materials with a Large Electrostatic Potential Difference. J. Am. Chem. Soc. 2019, 141 (19), 7743-7750.

31. Sun, Z.; Ye, Q.; Chi, C.; Wu, J., Low band gap polycyclic hydrocarbons: from closed-shell near infrared dyes and semiconductors to open-shell radicals. Chem. Soc. Rev. 2012, 41 (23), 7857-7889.

32. Zeng, Z.; Shi, X.; Chi, C.; Lopez Navarrete, J. T.; Casado, J.; Wu, J., Proaromatic and anti-aromatic pi-conjugated molecules: an irresistible wish to be diradicals. Chem. Soc. Rev. 2015, 44 (18), 6578-6596.

33. Li, Y.; Heng, W. K.; Lee, B. S.; Aratani, N.; Zafra, J. L.; Bao, N.; Lee, R.; Sung, Y. M.; Sun, Z.; Huang, K. W.; Webster, R. D.; Lopez Navarrete, J. T.; Kim, D.; Osuka, A.; Casado, J.; Ding, J.; Wu, J., Kinetically blocked stable heptazethrene and octazethrene: closed-shell or open-shell in the ground state? J. Am. Chem. Soc. 2012, 134 (36), 14913-14922.

34. Sun, Z.; Zeng, Z.; Wu, J., Zethrenes, extended p-quinodimethanes, and periacenes with a singlet biradical ground state. Acc. Chem. Res. 2014, 47 (8), 25822591.

35. Casado, J., Para-Quinodimethanes: A Unified Review of the Quinoidal-VersusAromatic Competition and its Implications. Top. Curr. Chem. 2017, 375 (4), 73.

36. Scharber, M. C.; Mühlbacher, D.; Koppe, M.; Denk, P.; Waldauf, C.; Heeger, A. J.; Brabec, C. J., Design Rules for Donors in Bulk-Heterojunction Solar CellsTowards 10\% Energy-Conversion Efficiency. Adv. Mater. 2006, 18 (6), 789-794.

37. Roncali, J.; Leriche, P.; Blanchard, P., Molecular materials for organic photovoltaics: small is beautiful. Advanced Materials 2014, 26 (23), 3821-38.

38. Du, T.; Gao, R.; Deng, Y.; Wang, C.; Zhou, Q.; Geng, Y., IndandioneTerminated Quinoids: Facile Synthesis by Alkoxide-Mediated Rearrangement Reaction and Semiconducting Properties. Angew. Chem. Int. Ed. 2020, 59 (1), 221-225. 39. Qu, J.; Chen, H.; Zhou, J.; Lai, H.; Liu, T.; Chao, P.; Li, D.; Xie, Z.; He, F.; Ma, Y., Chlorine Atom-Induced Molecular Interlocked Network in a Non-Fullerene Acceptor. ACS Appl. Mater. Interfaces 2018, 10 (46), 39992-40000.

40. Du, T.; Gao, R.; Deng, Y.; Wang, C.; Zhou, Q.; Geng, Y., IndandioneTerminated Quinoids:Facile Synthesis by Alkoxide-Mediated Rearrangement Reaction and Semiconducting Properties. Angew. Chem. Int. Ed. 2019, 1(59), 221.

41. Zeng, Z.; Ishida, M.; Zafra, J. L.; Zhu, X.; Sung, Y. M.; Bao, N.; Webster, R. D.; Lee, B. S.; Li, R. W.; Zeng, W.; Li, Y.; Chi, C.; Lopez Navarrete, J. T.; Ding, J.; Casado, J.; Kim, D.; Wu, J., Pushing extended p-quinodimethanes to the limit: stable tetracyano-oligo(N-annulated perylene)quinodimethanes with tunable ground states. $J$. Am. Chem. Soc. 2013, 135 (16), 6363-6371.

42. Wang, Q.; Li, M.; Peng, Z.; Kirby, N.; Deng, Y.; Ye, L.; Geng, Y., Calculation aided miscibility manipulation enables highly efficient polythiophene:nonfullerene photovoltaic cells. Sci. China Chem. 2021, 64, 478-487.

43. $\quad$ Pang, S.; Wang, Z.; Yuan, X.; Pan, L.; Deng, W.; Tang, H.; Wu, H.; Chen, S.; Duan, C.; Huang, F.; Cao, Y., A Facile Synthesized Polymer Featuring B-N Covalent Bond and Small Singlet-Triplet Gap for High-Performance Organic Solar Cells. Angew. Chem. Int. Ed. 2021, 60 (16), 8813-8817. 
44. Frisch, M.; Trucks, G.; Schlegel, H.; Scuseria, G.; Robb, M.; Cheeseman, J.; Scalmani, G.; Barone, V.; Petersson, G.; Nakatsuji, H. J. T. i. n. c. r. f. t. r., Gaussian 16, Revision A. 03, Gaussian, Inc., Wallingford CT, 2016. 2019.

45. Becke, A. D., A new mixing of Hartree-Fock and local density-functional theories. J. Chem. Phys. 1993, 98 (2), 1372-1377.

46. Stephens, P. J.; Devlin, F. J.; Chabalowski, C. F.; Frisch, M. J., Ab Initio Calculation of Vibrational Absorption and Circular Dichroism Spectra Using Density Functional Force Fields. J. Chem. Phys. 1994, 98 (45), 11623-11627.

47. Schuchardt, K. L.; Didier, B. T.; Elsethagen, T.; Sun, L.; Gurumoorthi, V.; Chase, J.; Li, J.; Windus, T. L., Basis Set Exchange: A Community Database for Computational Sciences. J. Chem. Inf. Model. 2007, 47 (3), 1045-1052.

48. Francl, M. M.; Pietro, W. J.; Hehre, W. J.; Binkley, J. S.; Gordon, M. S.; DeFrees, D. J.; Pople, J. A., Self-consistent molecular orbital methods. XXIII. A polarization-type basis set for second-row elements. J. Chem. Phys. 1982, 77 (7), 36543665 .

49. Yamaguchi, K., The electronic structures of biradicals in the unrestricted Hartree-Fock approximation. Chem. Phys. Lett. 1975, 33 (2), 330-335.

50. Schleyer, P. v. R.; Manoharan, M.; Wang, Z.-X.; Kiran, B.; Jiao, H.; Puchta, R.; van Eikema Hommes, N. J. R., Dissected Nucleus-Independent Chemical Shift Analysis of $\pi$-Aromaticity and Antiaromaticity. Org. Lett. 2001, 3 (16), 2465-2468.

51. Tomasi, J.; Mennucci, B.; Cammi, R., Quantum Mechanical Continuum Solvation Models. Chem. Rev. 2005, 105 (8), 2999-3094.

52. Geuenich, D.; Hess, K.; Köhler, F.; Herges, R., Anisotropy of the Induced Current Density (ACID), a General Method To Quantify and Visualize Electronic Delocalization. Chem. Rev. 2005, 105 (10), 3758-3772. 


\title{
Supporting Information
}

\section{Open-shell Donors and Closed-shell Acceptors in Organic Solar Cells}

\author{
Zhongxin Chen, ${ }^{1}$ Yuan Li, ${ }^{* 1}$ Wenqiang Li, ${ }^{1}$ Weiya Zhu, ${ }^{1}$ Miao Zeng, ${ }^{1}$ Jingwen Wang, ${ }^{2}$ Xiaojin \\ Zhang, ${ }^{1}$ Xiaobin Peng, ${ }^{1}$ Tian Du, ${ }^{3}$ Yunfeng Deng, ${ }^{3}$ Ren Zhang, ${ }^{4}$ Gongqiang Li ${ }^{4}$, Jianhui Hou ${ }^{2}$ \\ and Fei Huang*1
}

1 State Key Laboratory of Luminescent Materials and Devices, Institute of Polymer Optoelectronic Materials and Devices, South China University of Technology, Guangzhou, 510640, P. R. China.

2 Beijing National Laboratory for Molecular Sciences, State Key Laboratory of Polymer Physics and Chemistry, CAS. Research/Education Center for Excellence in Molecular Sciences, Institute of Chemistry, Chinese Academy of Sciences, Beijing 100190, P. R. China

3 Tianjin Key Laboratory of Molecular Optoelectronic Science, Tianjin. University and Collaborative Innovation Center of Chemical Science and Engineering (Tianjin), Tianjin 300072, P. R. China.

4 Institute of Advanced Materials, Nanjing Tech University, Nanjing 211816, P. R. China.

*Correspondence author: celiy@scut.edu.cn,hjhzlz@iccas.ac.cn, msfhuang@scut.edu.cn 


\section{Table of contents}

$\begin{array}{lr}\text { Figure S1. Molecular structures. } & 18\end{array}$

Figure S2. Variable temperature ${ }^{1} \mathrm{H}$ NMR spectra of BTR 19

Figure S3. The superconducting quantum interference device spectrum of PTB7-Th. 20

Figure S4. Optimized molecular structures and electrostatic potential distributions. $\quad 20$

Figure S5. Optimized geometric parameters and $\operatorname{NICS}_{\text {iso }}(1)(\mathrm{ppm})$ values 21

$\begin{array}{ll}\text { Figure S6. EQE curves of the OSCs. } & 21\end{array}$

Table S1. Device performance of IDIC and IDI. 21

Figure S7. Numerical trend of nucleus independent chemical shift values. 22

Figure S8. The anisotropy of the induced current density plots. 22

Figure S9. The solar cell performance of OSCs containing PTB7-Th and DPPC-IC4F. 23 


\section{Results}

Molecular structures

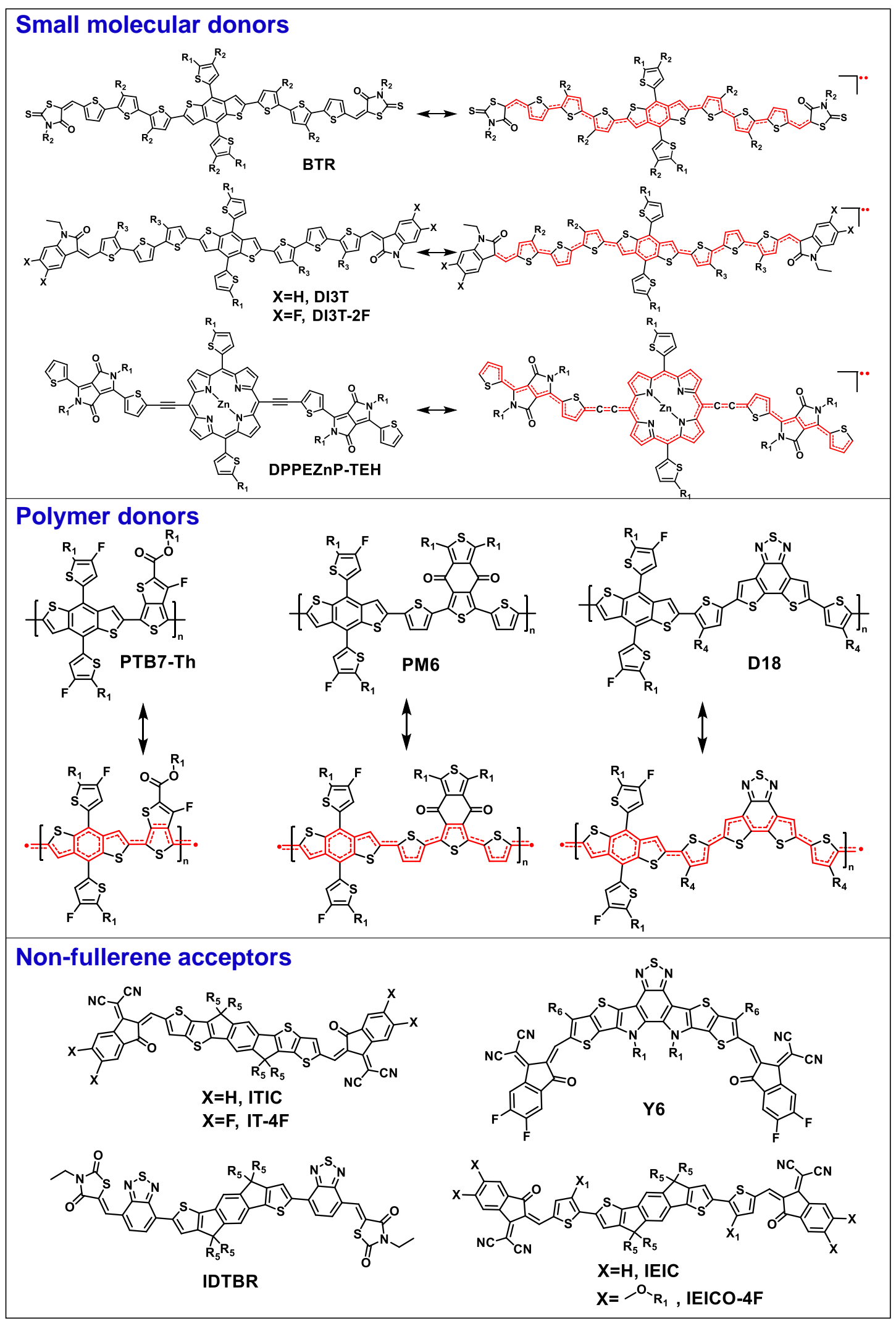

Figure S1. Molecular structures of small molecular donors, polymer donors, and non-fullerene acceptors. 


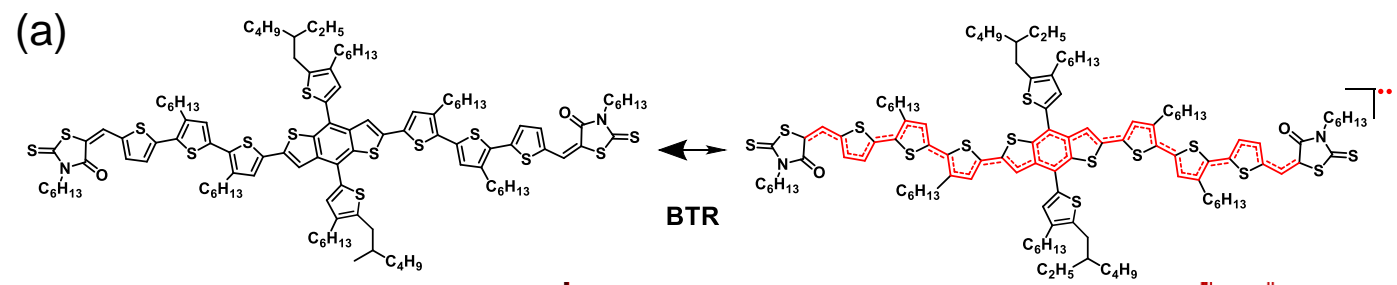

(b)

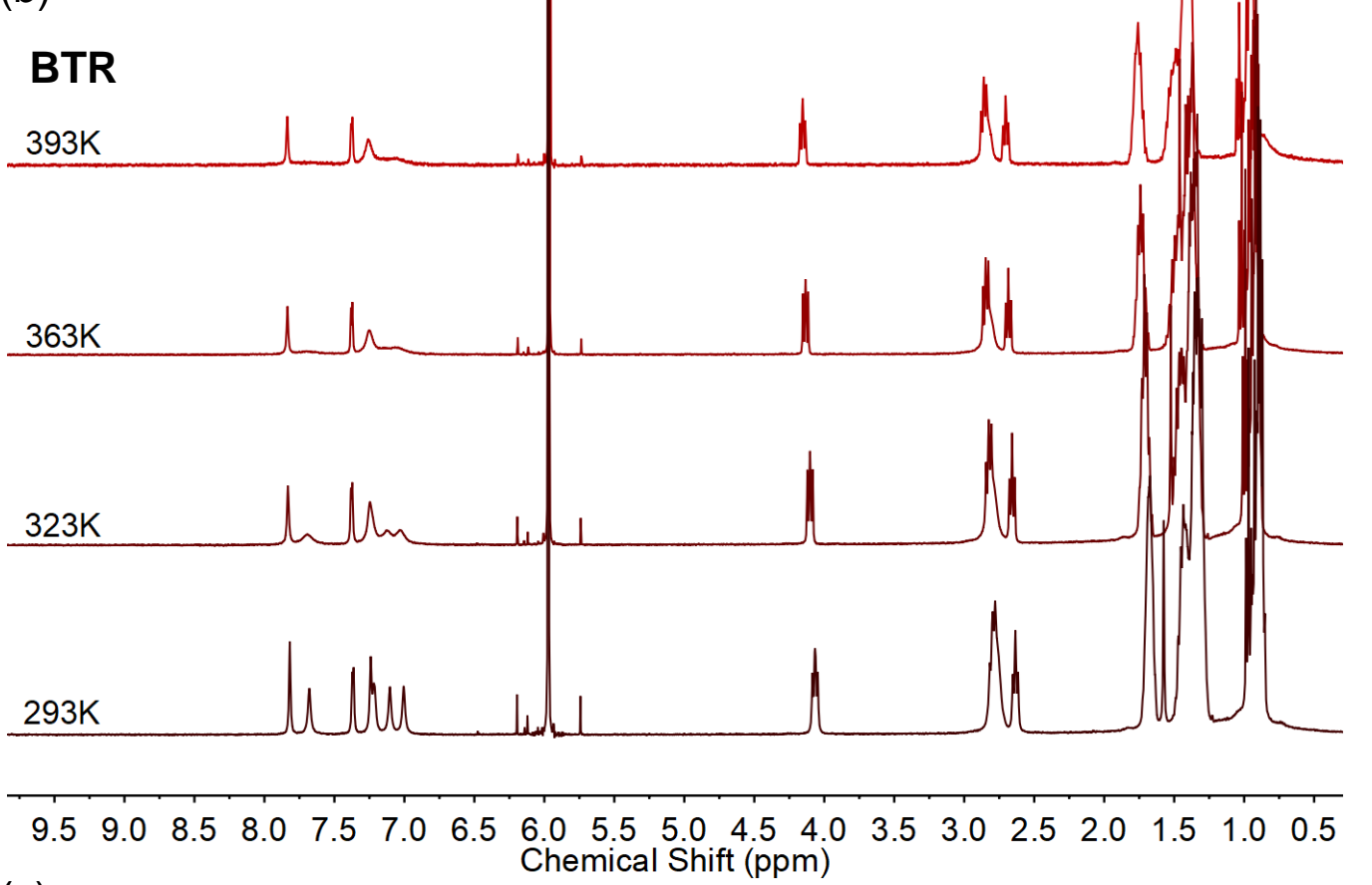
(c)
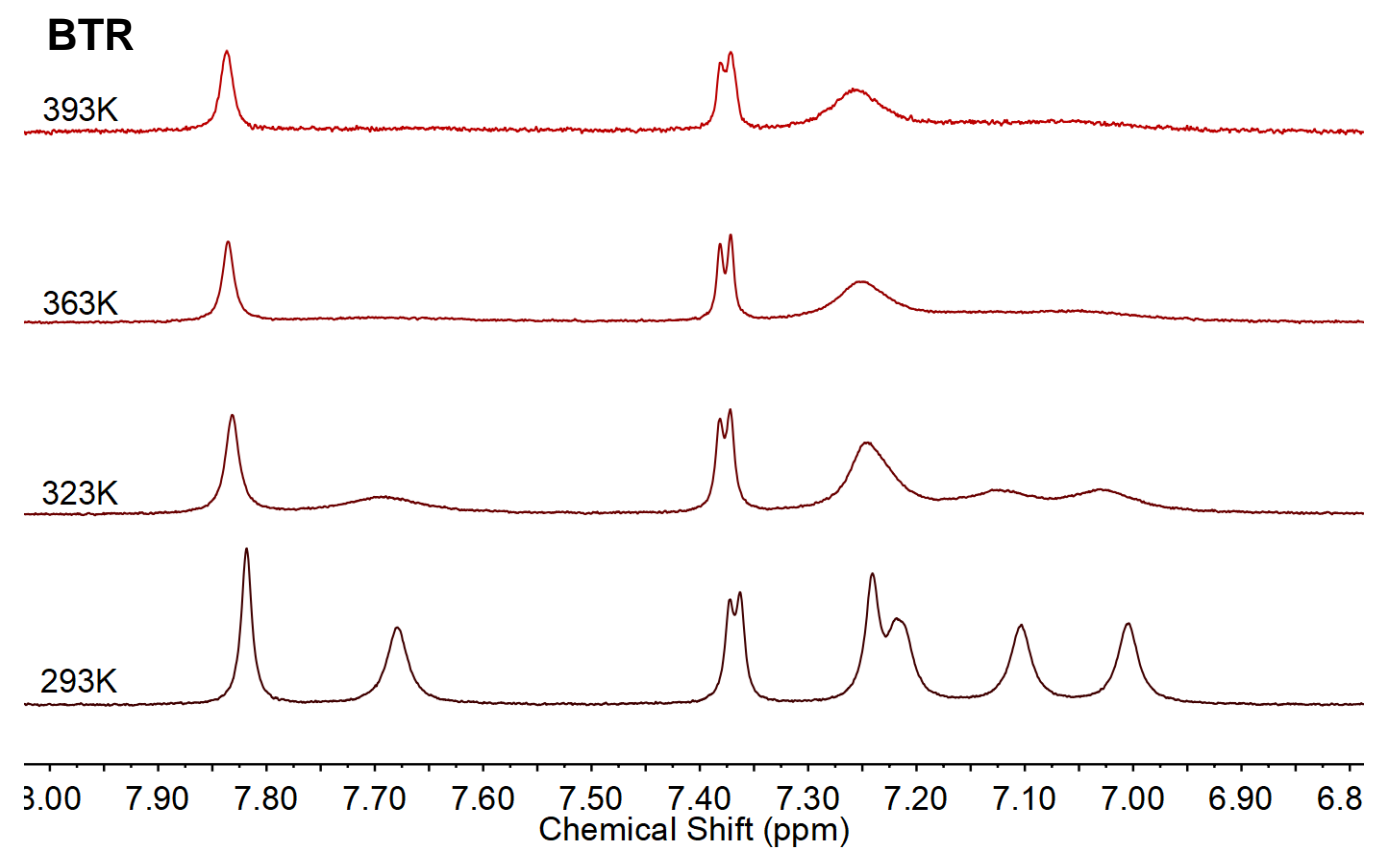

Figure S2. (a) Resonance structures of BTR. (b) Variable temperature ${ }^{1} \mathrm{H}$ NMR spectra and (c) the enlarged spectra of BTR in the aromatic region (6.8 to $8.0 \mathrm{ppm}$ ) in $\mathrm{C}_{2} \mathrm{D}_{4} \mathrm{Cl}_{4}$ in the temperature range from 293 to $393 \mathrm{~K}$. 


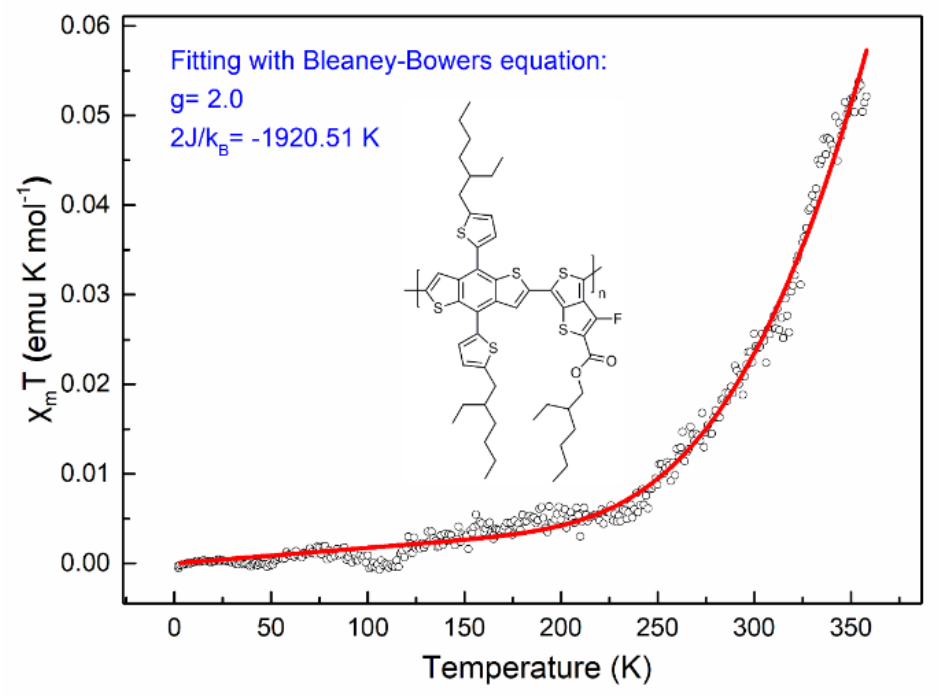

Figure S3. The SQUID spectrum of PTB7-Th. The curve fits with Bleaney-Bowers equation and the calculated $2 J / k_{B}$ are inset in the figure.

(a)
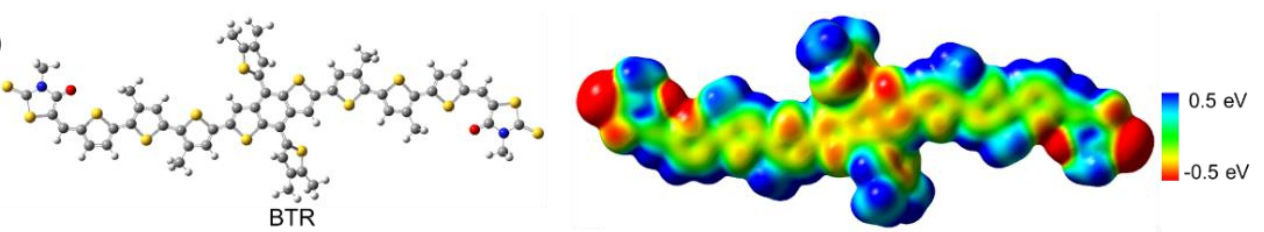

(b)

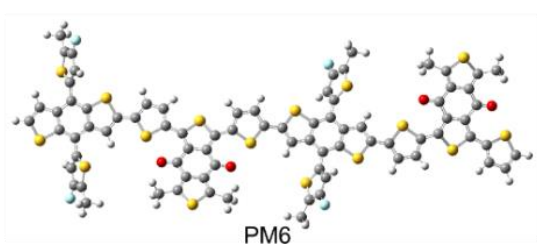

(c)
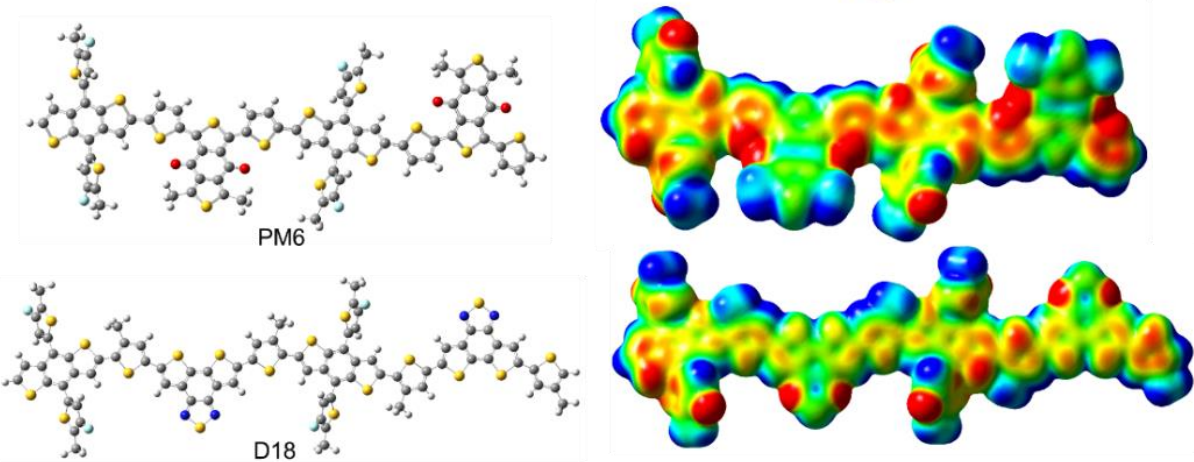

(d)
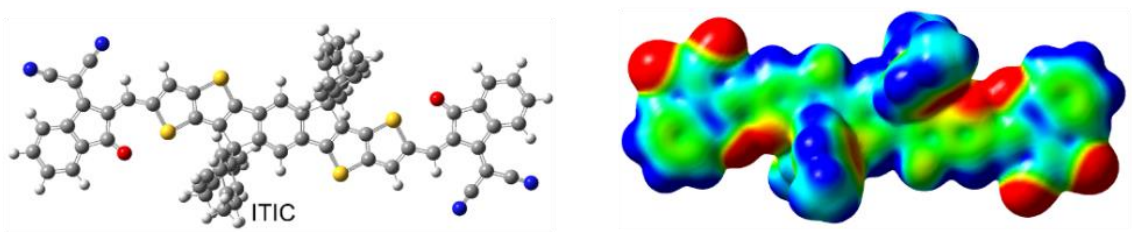

(e)
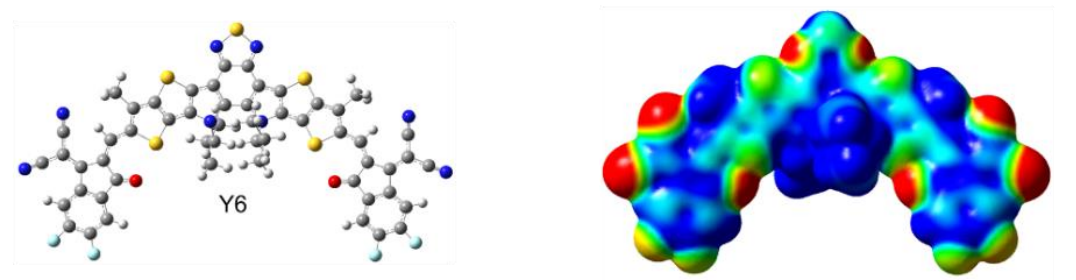

Figure S4. Optimized molecular structures and electrostatic potential (ESP) distributions of (a) BTR, (b) PM6 (two repeating units) (c) D18 (two repeating units), (d) ITIC, and (e) Y6, respectively. 

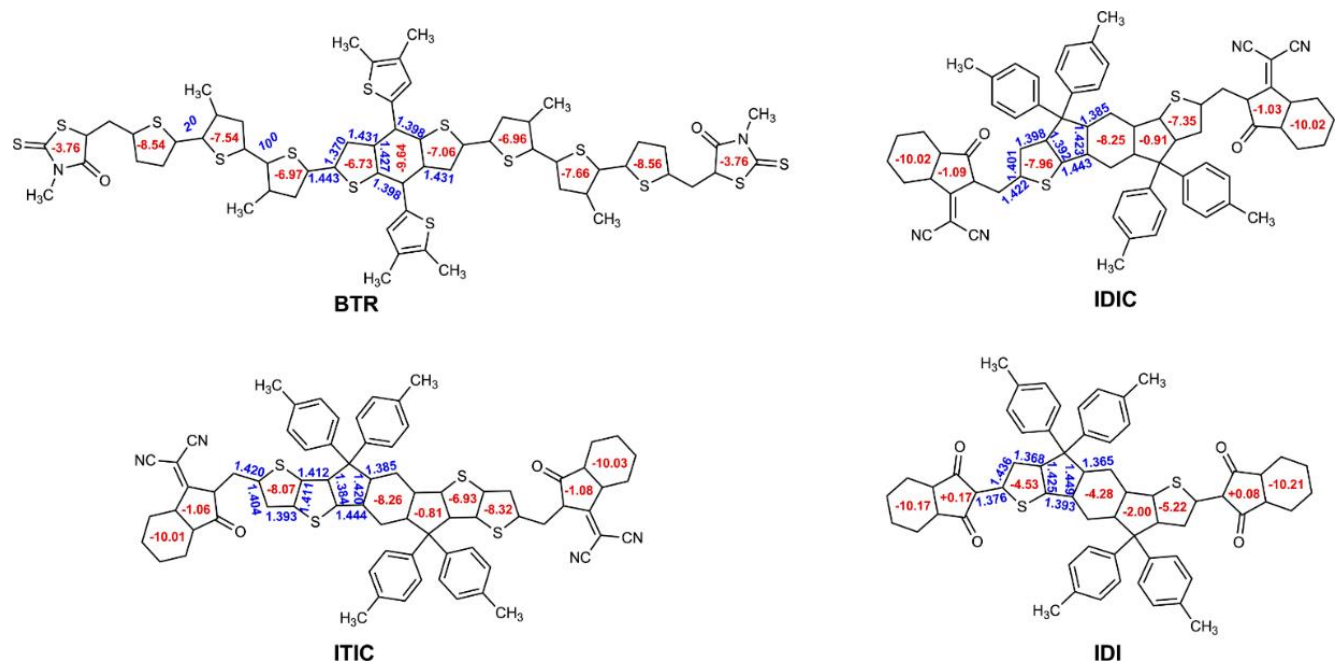

Figure S5. Optimized geometric parameters and NICS $_{\text {iso }}(1)(\mathrm{ppm})$ values of BTR, ITIC, IDIC, and IDI calculated at B3LYP/6-31G $(d, p)$ level of theory and basis set. Bond lengths are provided in $\AA$.

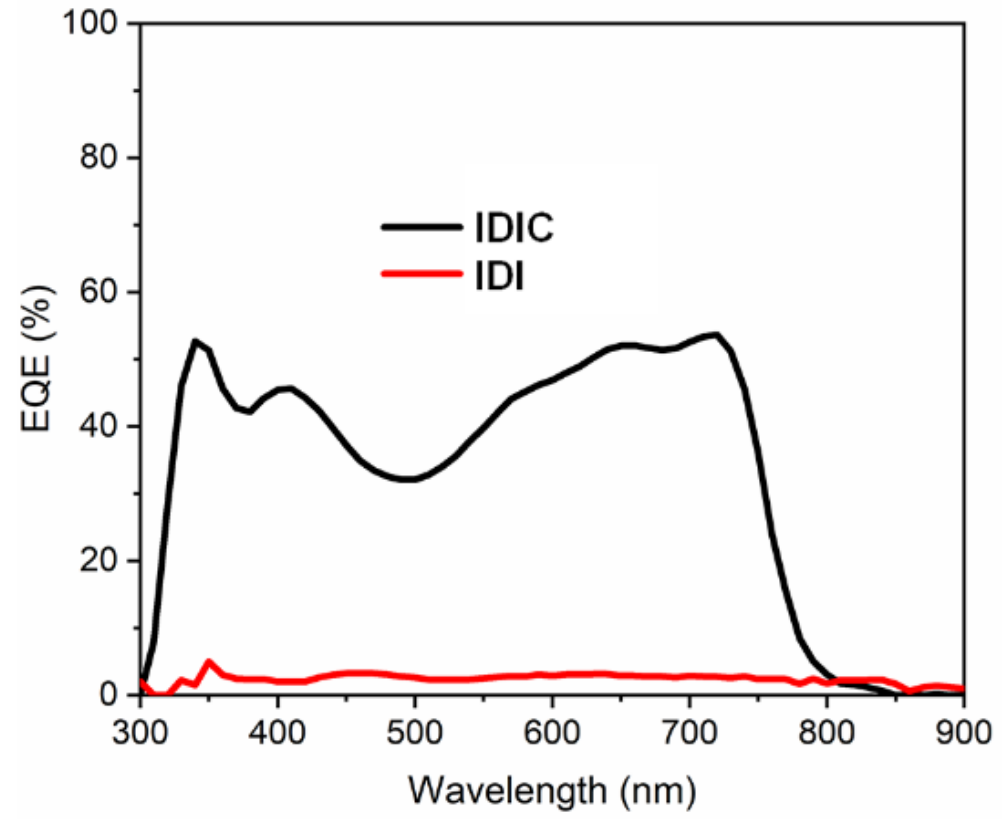

Figure S6. EQE curves of OSCs based on PTB7-Th/IDIC and PTB7-Th/IDI, respectively.

Table S1. Device performance of IDIC and IDI. The device based on structure: ITO/PEDOT: PSS/PTB7-Th: Acceptor/PFN-Br/Ag. The donor/acceptor ratio= 1: $1(12 \mathrm{mg} / \mathrm{mL}$ in total $)$. The processing solvent is chloroform.

\begin{tabular}{ccccccc}
\hline & $\mathrm{N}(\mathrm{rpm})$ & $V_{\mathrm{oc}}(\mathrm{V})$ & $J_{\mathrm{sc}}\left(\mathrm{mA}^{*} \mathrm{~cm}^{-2}\right)$ & $\mathrm{FF}(\%)$ & $\mathrm{PCE}(\%)$ & $\mathrm{PCE}_{\max }(\%)$ \\
\hline IDIC & 2400 & $0.78 \pm 0.01$ & $12.60 \pm 0.10$ & $51.52 \pm 0.83$ & $5.09 \pm 0.03$ & 5.13 \\
IDI & 2400 & $0.34 \pm 0.01$ & $0.93 \pm 0.03$ & $36.66 \pm 1.47$ & $0.11 \pm 0.01$ & 0.13 \\
\hline
\end{tabular}



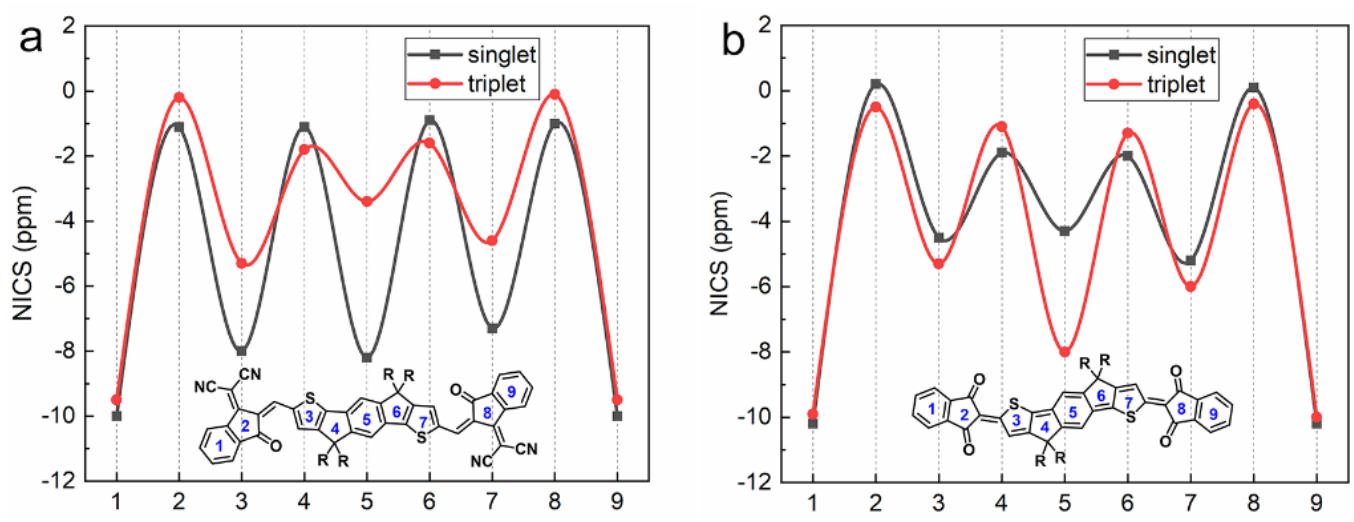

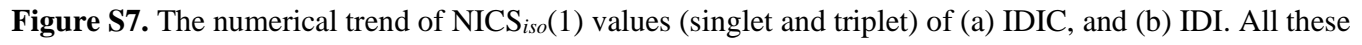
molecules show an increase of aromaticity from singlet to triplet state.

(a)

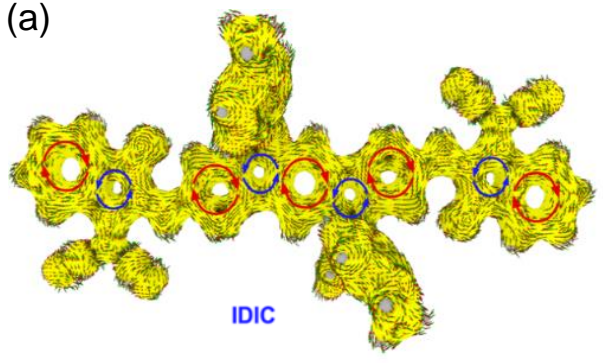

(c)

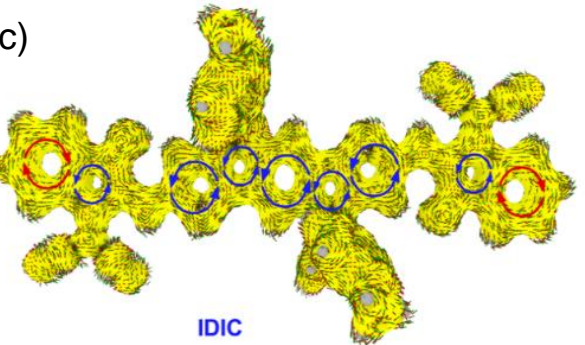

(b)

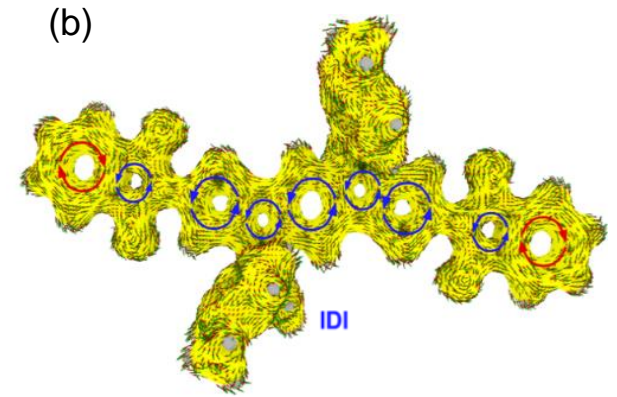

(d)

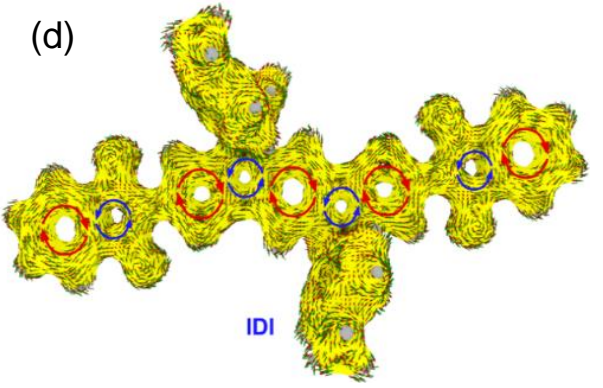

Figure S8. AICD plots for IDIC and IDI in their singlet state (a, b) and triplet state (c, d), respectively, at B3LYP/6$31 \mathrm{G}(d, p)$ level of theory and basis set. The red and blue arrows indicate clockwise (diatropic: aromatic) and counterclockwise (paratropic: quinoidal) ring current, respectively. The applied magnetic field is perpendicular to the molecular backbone and points out through the plane of the paper. AICD plots generated with an isovalue $=$ $0.025 \mathrm{au}$. 
(a)
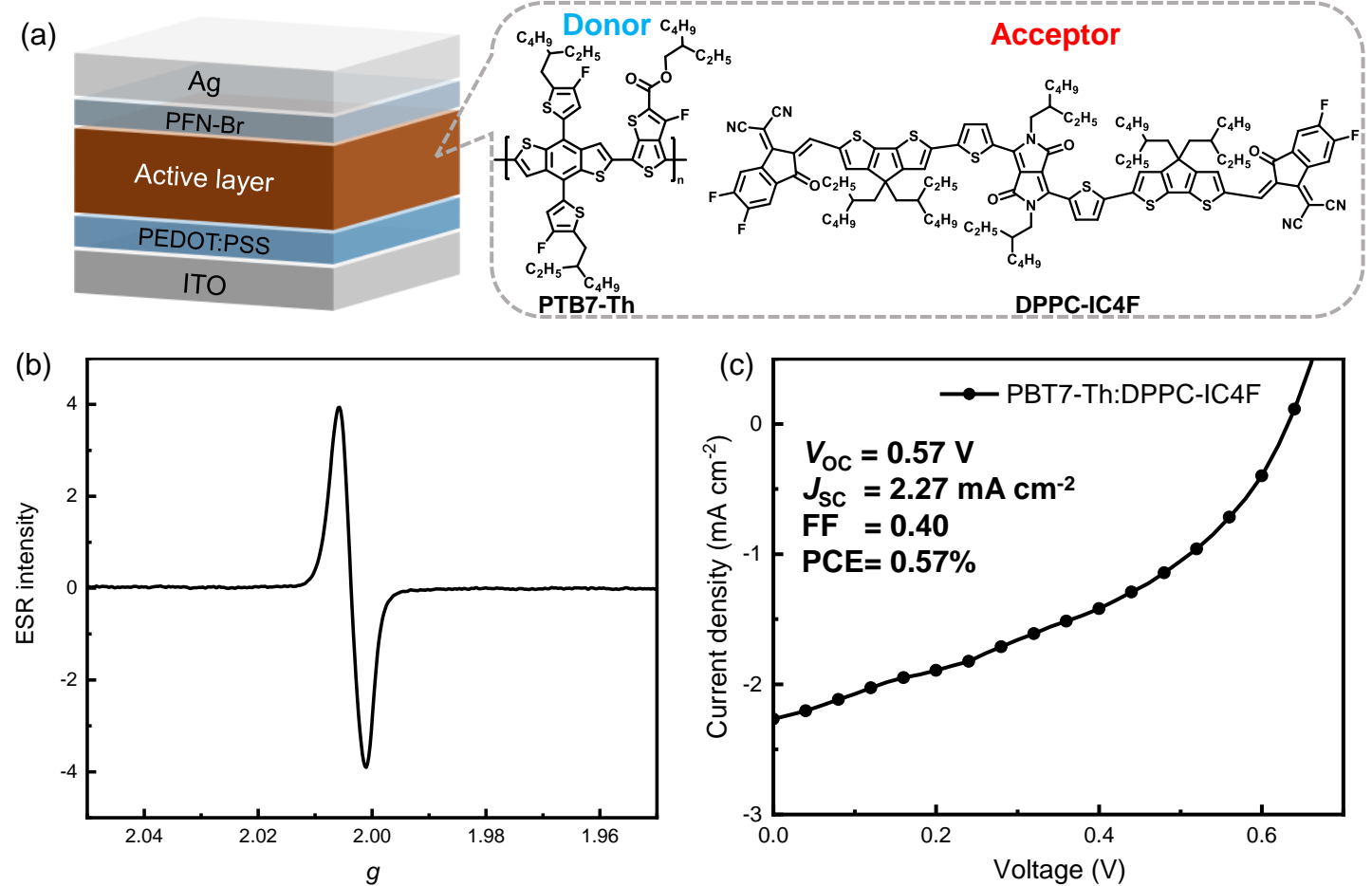

Figure S9. The solar cell performance of OSCs containing (a) PTB7-Th and (b) DPPC-IC4F. (c) The J-V curve of the OSC device. The device based on structure: ITO/PEDOT: PSS/PTB7-Th: DPPC-IC4F/PFN$\mathrm{Br} / \mathrm{Ag}$. The donor/acceptor ratio $=1: 1.5(20 \mathrm{mg} / \mathrm{mL}$ in total $)$. The processing solvent is chlorobenzene. 